\title{
Vilazodone for the Treatment of Paternal Post Natal Depression
}

\section{Hani Raoul Khouzam*}

Medical Director, Employee Behavioral Health Dartmouth - Hitchcock Medical Center, Professor of Psychiatry, The Geisel School of Medicine at Dartmouth, Hanover, New Hampshire, USA

\begin{abstract}
Maternal postpartum depression is well recognized by clinicians as one of the psychiatric complication of delivery, conversely paternal postpartum depression or paternal postnatal depression which affects up to $10 \%-14 \%$ of new fathers 6 to 12 months after a child birth is rarely recognized and treated. Without proper diagnosis and prompt treatment paternal postnatal depression could lead to dire consequences including, deterioration in paternal mental health, loss of paternal bonding with the newborn child, which has been associated with long term later child psychiatric complications such as conduct disorders, hyperactivity, anxiety, depression, posttraumatic stress disorder and delays in language acquisition. It can also lead to marital conflicts, stressful family interactions and psychiatric disability leading to potential loss of income, and increase medical care costs. Like major depressive disorders the treatment of paternal postnatal depression may require psychopharmacological treatment typically with selective serotonin reuptake inhibitors or serotonin norepinephrine reuptake inhibitors. The purpose of this report is to increase clinicians awareness in recognizing and treating paternal postnatal depression as illustrated in a case of a 30 year-old new father who developed paternal postnatal depression and his progress in response to treatment with the first-of-class serotonin partial agonist reuptake inhibitor antidepressant vilazodone which basic pharmacology is summarized.
\end{abstract}

Keywords: Parental postnatal depression; Postpartum depression; Serotonin partial agonist reuptake inhibitor; Vilazodone; Treatment.

\section{Introduction}

Despite its common occurrence affecting up to $10 \%-14 \%$ of new fathers 6 to 12 months after a child birth [1]; paternal postnatal (PPND) is not usually recognized or treated [2,3]. Like major depressive disorder (MDD) the treatment of PPND may require the combination of psychopharmacology, psychotherapy, social and spiritual intervention [3]. Most clinical guidelines recommend cognitive behavioral therapy (CBT) as the psychotherapy of choice alone or combined with selective serotonin reuptake inhibitors (SSRIs) or serotonin norepinephrine reuptake inhibitors (SNRIs) for the treatment of MDD. The presence of maternal postpartum depression (MPPD) [4] and marital discord [5] are significantly correlated with PPND. This report describes a case of a 30 year-old new father who developed PPND in the absence of both MPPD and marital discord who did not tolerate treatment with the SSRI fluoxetine and the SNRI venlafaxine but had a favorable response to vilazodone the first-of-class of antidepressants classified as a serotonin partial agonist reuptake inhibitor (SPARI). The patient initial clinical diagnosis of PPND was confirmed by using the Diagnostic and statistical manual of mental disorders 5 th edition (DSM-5) criteria for MDD [6]. The patient's presenting symptoms and clinical progress were monitored using the Patient Health Questionnaire (PHQ)-2 [7], PHQ-9 [8] and the 17-item Hamilton Rating Scale for Depression (HAM-D-17) [9].

\section{Case Presentation}

Mr. I, was a 30 year-old gentleman whose firstborn son was delivered following a 9 month of uneventful pregnancy and labor. His wife was immensely thankful to have a perfect healthy newborn boy and nick named him "Sunny" to reflect his joyful and calm temperament. Mr. I, returned to work after 5 weeks of family leave and was relatively able to balance his new role as a father with his profession as an architect. He was overjoyed with his loving wife, his supportive parents and parents in-laws. He was frequently contacted via social media by his close friends and distant relatives who took turns in sending gifts to the whole family including his wife and Sunny. Two weeks later, Mr. I, could not experience any feelings for his son He would often go for many days without looking at him and rarely helping his wife in changing his diaper or bottle feeding him. He felt sad, hopeless, irritable, anxious, and angry for no apparent reasons. He also lost interest in his work and procrastinated on pending architectural designs. This was an unusual pattern of behaviors, since he has always been known for his punctuality and timely completion of his assigned projects. He lost all interests in intimate relationship and avoided sleeping in the same bed with his wife. Although he felt extremely tired, he could not sleep. He was unable to concentrate and reported "feelings guilt and shame for not being connected to his son " and was convinced that he was not a good father and that he was failing his wife and his son "Sunny". He began to have disturbing thoughts that "everyone would be better off without him". These statements alarmed his wife and his parents and he was persuaded to seek professional help.

\section{Screening and Diagnostic Tools}

The Patient Health Questionnaire (PHQ)-2 [7] yielded an elevated score of 6, which was then followed by the administration of the PHQ-9 [8] which showed a high score of 25 . The presenting symptoms met the diagnostic and statistical manual of mental disorders 5th edition (DSM5) criteria of a severe MDD [6]. The 17-item Hamilton Rating Scale for Depression (HAM-D-17) [9] confirmed the severity of the clinical

*Corresponding author: Khouzam H, Medical Director, Employee Behavioral Health Dartmouth - Hitchcock Medical Center, Professor of Psychiatry, The Geisel School of Medicine at Dartmouth, Hanover, New Hampshire, USA, Tel: 603.650.4725; E-mail: Hani.R.Khouzam@Dartmouth.edu

Received: November 02, 2015; Accepted: November 03, 2015; Published: Novomber 15, 2015

Citation: Khouzam HR (2015) Vilazodone for the Treatment of Paternal Post Natal Depression. Clin Depress 1: 104.

Copyright: (c) 2015 Khouzam HR. This is an open-access article distributed under the terms of the Creative Commons Attribution License, which permits unrestricted use, distribution, and reproduction in any medium, provided the original author and source are credited. 
presentation reflecting a score of 25 . To rule out primary medical illness standard laboratory tests were ordered and were all normal.

\section{History and Physical Examination}

Mr. I, did not use any alcohol or illicit drugs, he also did not smoke or drink any caffeinated beverages. He had no past history of psychiatric or substance use disorders and there was no family history of psychiatric conditions. He had a completely normal physical examination and was not taking any prescribed or over the counter medications.

\section{Treatment}

Mr. I, agreed to attend a weekly "New Fathers" peer support group, and weekly CBT sessions and to consider treatment with an antidepressant. He was reluctant to take any kind of medication, however he realized that he had no emotional energy and could not attend the peers support group meetings and he had no motivation to participate in CBT. He agreed to consider treatment with the SSRI fluoxetine at the dose of $20 \mathrm{mg}$ once a day which he took for 1 week and stopped it due to intolerable nausea and headaches. He did not want to consider another SSRI such as sertraline, paroxetine or citalopram, but agreed to take the SNRI venlafaxine at the dose of $37.5 \mathrm{mg}$ once a day, however he experienced drowsiness, dizziness, feeling of nervousness and constipation and adamantly refused to consider another SRNI such as duloxetine. Vilazodone which is a serotonin partial agonist reuptake inhibitor (SPARI) was then started at the dose of $10 \mathrm{mg} /$ day for 7 days without side effects, followed by $20 \mathrm{mg} /$ day for 7 days without side effects, then increased to $40 \mathrm{mg} /$ day.

Mr. I. was satisfied with the absence of any adverse effects and wanted to continue treatment for few weeks to determine if the medication is helpful. He took vilazodone at the dose of $40 \mathrm{mg} /$ day for 4 weeks and was expressing feelings of disappointment about the lack of any beneficial effects, but agreed to maintain the treatment. On week 6 he noticed a marked improvement in his feelings toward his son "Sunny" and his wife and was eager to return to work. He also was able to engage in social interaction without irritability and resumed sleeping in the same bed with his wife and began to enjoy their intimate relationship. In his follow-up psychiatric assessment he had negative PHQ -2 and PHQ-9 scores [7,8] and a HAM-D-17 score of 6 [9]. He was cleared to resume his employment with his architectural firm. He did not feel comfortable lowering or discontinuing vilazodone, and began attending the "New Fathers" peers support group but could not afford attending the CBT weekly therapy sessions. It has been now 4 months since the initiation of vilazodone therapy and Mr. I, continues to be free of any PPND symptoms. At the time of writing this report he continues to take vilazodone at the dose of $40 \mathrm{mg} /$ day without adverse effects. He has also assumed a leadership role in the 'New Fathers' peers support group. He has received a special achievement award as an outstanding and creative architect and is overwhelmed with joy because his wife was pregnant and they are anticipating the birth of their second child a baby girl.

\section{Discussion}

Multiple studies suggest that PPND is rarely diagnosed or treated and as a result it has a variable reported incidence of $4 \%$ to $25 \%$ within the first 12 months of a child birth [4,10-12]. Without treatment PPND could lead to dire consequences including, deterioration in paternal mental health, loss of paternal bonding with the newborn child, which has been associated with long term later child psychiatric complications such as conduct disorders, hyperactivity, anxiety, depression, posttraumatic stress disorder and delays in language acquisition [4,11,13-17]. PPND can also lead to marital conflicts, stressful family interactions, financial burden due to potential loss of income, and increase medical care costs $[4,11,17]$.The presentation and causes of PPND are different from MPPD .Hormonal changes were suggested as contributing factors to MPPD, whereas sudden and unexpected lifestyle changes are thought to trigger fathers' PPND. There is a moderate, positive correlation between the incidence of PPND and MPPD $[18,19]$. Some reports also suggest that MPPD and marital discord could trigger PPND [20]. In Mr. I, case these two risk factors were not present, and his negative personal and family history of depression suggest that this first onset of MDD was indeed a PPND related to the birth of his first child Sunny. Fathers with PPND-like men with MDD in general-are more likely than depressed women to engage in destructive behaviors, including alcohol or drug abuse, angry outbursts, or taking unnecessary risks such as reckless driving or extramarital infidelity and fortunately Mr. I did not exhibit these risky behaviors, however he developed a depressed mood, loss of interest in pleasurable activities, insomnia, restlessness, fatigue, guilt, impaired concentration, and thoughts of a worthless life.

Screening for depression is available using various screening tools such as the PHQ-2 [7] and the PHQ-9 [8]. In adults; the PHQ-2 has a $97 \%$ sensitivity and $67 \%$ specificity in identifying the presence of a clinical depression [21]. The severity of Mr. I, PPND was demonstrated with his positive total score of 6 on the PHQ-2 [7], which warranted further assessment with the PHQ-9 [8]. The diagnostic validity and the reliability of the PHQ-9 has long been established and scores $>10$ had a sensitivity of $61 \%$ and a specificity of $94 \%$ for MDD. Individuals who scored high $(\geq 10)$ on the PHQ-9 are between 7 to 13.6 times more likely to be diagnosed with depression by mental health professional. On the other hand, individuals scoring low $(\leq 4)$ on the PHQ-9 had a less than a 1 in 25 chance of having depression [8]. A PHQ-9 score of 1 to 4 suggests minimal depression, a score 5 to 9 is indicative of mild depression, a score 10 to 14 is equivalent to a moderate depression, a 15 to 19 score is an indication of a moderately severe depression, a score of 20 to 27 is considered a strong indication for a severe depression. Mr. I, PHQ-9 revealed a positive total score of 25 suggesting severe depression due PPND.

Mr. I, also met the DSM-5 diagnostic criteria of a severe major depressive episode [6] which was manifested by his sad mood, marked loss of interest in virtually all activities, insomnia, fatigue, loss of energy, feelings of guilt, diminished ability to think or concentrate, and disturbing thoughts that "everyone would be better off without him". The administration of the HAM-D-17 was added to his overall evaluation due to its proven usefulness in determining the level of depression before, during, and after treatment. It has $86.4 \%$ sensitivity and $92.2 \%$ specificity: Although the HAM-D form lists 21 items, the scoring is based on the first 17 items [9]. Eight items are scored on a 5 -point scale, ranging from $0=$ not present to $4=$ severe. Nine are scored from 0-2. A HAM-D-17 score of 0-7 is considered normal, a score of 8-13 suggest mild depression, a score of $14-18$ is related to a moderate depression, while a score of 19-22 has been associated with severe depression and a score above 22 is suggestive of a very severe depression [22]. Based on Mr.I, clinical presentation and psychiatric assessment his PPND met the criteria of a severe MDD requiring immediate and prompt treatment. As recommended by many clinical guidelines a combination of psychotherapy and psychopharmacology was suggested, but he could not initially attend the "New Fathers" peers support group or enroll in CBT. He also could not tolerate the SSRI fluoxetine and the SNRI venlafaxine but tolerated and responded well to the SPARI vilazodone. 
Vilazodone (Viibryd) is an oral antidepressant that is unrelated to tricyclic or tetracyclic antidepressants which was approved for the treatment of adults MDD in January 2011 [23]. It is the first-of-class of antidepressants which is classified as a serotonin partial agonist reuptake inhibitor (SPARI). Although its exact mechanism of action is not fully understood, it combines serotonin transporter (SERT) inhibition with a second property of serotonin 5HT 1A partial agonist and reuptake inhibition [24]. Generally, the therapeutic effect of serotonin reuptake inhibitors is believed to occur via blockade of the reuptake of serotonin at the presynaptic neuronal membrane and the chronic exposure to increased serotonin levels in the central nervous system(CNS) downregulates postsynaptic neuronal receptor binding sites, which may also contribute to antidepressant effects [24,25]. The combination of serotonin reuptake inhibition with $5 \mathrm{HT} 1 \mathrm{~A}$ partial agonism has long been known by clinicians to enhance the antidepressant properties and tolerability in some patients who did not tolerate SSRIs or SNRIs such as in the case of Mr. I $[26,27]$. SPARI actions could hypothetically lead to more antidepressant efficacy than selective SERT inhibition, and lesser sexual dysfunction due to lesser degrees of SERT inhibition than SSRIs. However these advantages have only been demonstrated in placebo controlled trials, but not in head-to-head trials of vilazodone against the SSRIs or the SNRIs [23-27]. Thus, there are many theoretical advantages to the SPARI combined mechanism, but proof of that advantage over current antidepressants or well-known combinations of two agents to achieve the same pharmacologic actions is still awaiting more clinical trials.

The safety profile and tolerability of vilazodone is comparable to other SSRIs and its main side effects include diarrhea, nausea, and headache [28]. It has less incidence of sexual side effects. Vilazodone is extensively metabolized in the liver; therefore, caution is advisable during its use in patients with hepatic disease, but no dosage adjustments are recommended in the presence of mild, moderate, or severe hepatic impairment $[28,29]$. Vilazodone is contraindicated for concomitant use with monoamine oxidase inhibitors (MAOIs) or within 14 days of stopping or starting an MAOI. Vilazodone is contraindicated in patients taking CYP3A4 inhibitors such as ketoconazole and topiramate which may lead to an increased vilazodone concentrations resulting into concentration-dependent adverse effects.3 Patients receiving certain medications affecting CYP450 metabolism such as topiramate or CYP3A4 inducers such as rifampin might result in a reduction in vilazodone levels leading to lack or loss of efficacy and may require dose adjustment. Similar to other antidepressants, vilazodone labeling carries warnings about serotonin syndrome, seizures, abnormal bleeding, activation of mania/hypomania, and hyponatremia [29].

Suicidal ideation and suicide attempt have been reported during post-marketing use of vilazodone. Suicidal ideation and behaviors have also been reported in other antidepressant clinical trials. A causal role has been established for antidepressants in inducing suicidality in children and young adults (18 to 24 years of age) during the early phase of treatment. Symptoms including aggressiveness, akathisia (psychomotor restlessness), agitation, anxiety, insomnia, irritability, hostility, mania, hypomania, impulsivity, and panic attacks have been reported in adult and pediatric patients being treated with antidepressants for major depressive disorder as well as for other indications, both psychiatric and nonpsychiatric. Although a causal link between the emergence of such symptoms and either the worsening of depression and/or the emergence of suicidal impulses has not been established, there is concern that such symptoms may represent precursors to emerging suicidality [29].

The dosing guidelines for vilazodone recommend titration over 2 weeks to achieve a target of an oral dose of $20 \mathrm{mg}-40 \mathrm{mg}$ to be taken once daily with food. The initial dose is $10 \mathrm{mg}$ once daily with food for 7 days, then $20 \mathrm{mg}$ once daily with food for 7 days. The dose may be increased up to $40 \mathrm{mg}$ once daily with food after a minimum of 7 days between dosage increases. Generally, acute episodes of depression require several months of continued pharmacologic therapy; however, patients should be assessed periodically to determine dosage requirements and the need for ongoing maintenance treatment. Steady state is usually achieved when patients have been on the stable target dose for approximately 2.5 weeks [29].

\section{Conclusion}

Depressive disorders constitute a major worldwide public health problem, with massive economic and quality-of-life consequences [30]. Prolonged, untreated PPND is associated with adverse behavioral, cognitive and emotional outcomes for not just the father or the mother but also the offspring [4,11,13-17,31]. The mental health of new fathers is an important issue to be addressed especially in those who develop PPND. This case report suggests that vilazodone the first member of SPARIs class of antidepressant medications can be used in the treatment of PPND in patients who do not respond to either an SSRI or an SNRI. Subsequent rando mized double blind placebo controlled studies would be needed to confirm vilazodone's efficacy in treating PPND.

\section{Acknowledgments}

To my wife Lynn, and children Andrea, Andrew and Adam, my sisters Hoda and Héla,my brother Hadi and my colleagues at Dartmouth-Hitchcock Medical Center Drs. William C.Torrey and Alan I. Green in Lebanon, New Hampshire and Drs.Robert W. Hierholzer and Avak A.Howsepian at UCSF in Fresno, California for their support and encouragement.

\section{References}

1. Kim P, Swain J (2007) Sad dads; paternal postpartum depression. Psychiatry 4: 35-47.

2. Ramchandani PG, Stein A, O'Connor TG, Heron J, Murray L, et al. (2008) Depression in men in the postnatal period and later child psychopathology: a population cohort study. J Am Acad Child Adolesc Psychiatry 47: 390-398.

3. Khouzam HR (2007) Depression: Guidelines for Effective Primary Care, Part 2 Treatment. Consultant 47: 841-848.

4. Paulson JF, Bazemore SD (2010) Prenatal and postpartum depression in fathers and its association with maternal depression: a meta-analysis. JAMA 303: 1961-1969.

5. Gawlik S, Muller M, Hoffmann L, Dienes A, Wallwiener M, et al. (2014) Prevalence of paternal perinatal depressiveness and its link to partnership satisfaction and birth concerns. Arch Womens Ment Health 17: 49-56.

6. American Psychiatric Association (2013) Diagnostic and statistical manual of mental disorders. 5th edition American Psychiatric Publishing; Arlington.

7. Spitzer RL, Kroenke K, Williams JB (1999) Validation and utility of a self-report Version of PRIME-MD: the PHQ Primary Care Study. JAMA 282: 1737-1744.

8. Kroenke K, Spitzer RL, Williams JB (2001) The PHQ-9: validity of a brief depression severity measure. Journal of General Internal Medicine 16: 606613.

9. Hamilton M (1960) A rating scale for depression. Journal of Neurology Neurosurgery and Psychiatry 23: 56-62.

10. Goodman JH (2104) Paternal postpartum depression, its relationship to maternal postpartum depression, and implications for family health. J Adv Nurs. 45: 26-35.

11. Ramchandani P, O'Connor TO, Evans J, Heron J, Murray L, et al. (2008) The effects of pre- and postnatal depression in fathers: A natural experiment comparing the effects of exposure to depression in offspring. The Journal of Child Psychology and Psychiatry 49: 1069-1078.

12. Escribà-Agüircorresponding $V$ Artazcoz L (2011) Gender differences in postpartum depression: a longitudinal cohort study. J Epidemiol Community 
Health. 65: 320-326.

13. Paulson J, Dauber S, Leiferman J (2006) Individual and combined effects of postpartum depression in mothers and fathers on parenting behavior. Pediatrics 118: 659-668.

14. Paulson J, Keefe H, Leiferman J (2009) Early parental depression and child language development. The Journal of Child Psychology and Psychiatry 50: 254-262.

15. Pilyoung K, Swain J (2007) Sad dads. Psychiatry 4: 35-47.

16. Radloff LS (1977) The CES-D scale: A self report depression scale for research in the general population. Applied Psychological Measurement 1: 385-401.

17. Ramchandani P, Stein A, Evans J, O'Connor T, ALSPAC Study Team (2005) Paternal depression in the postnatal period and child development: $A$ prospective population study. The Lancet 365: 2201-2205.

18. Dennis C L, Letourneau N (2007) Global and relationship specific perceptions of support and the development of postpartum depressive symptomatology. Social Psychiatry and Psychiatric Epidemiology 42: 389-395.

19. Goodman J (2104) Paternal postpartum depression, its relationship to maternal postpartum depression, and implications for family health. Journal of Advanced Nursing 45: 26-35.

20. Halle C, Dowd T, Fowler C, Rissel K, Hennessy K, et al. (2008) Supporting fathers in the transition to parenthood. Contemporary Nurse 31: 57-70.

21. Gilbody S, Richards D, Brealey S, Hweitt C (2007) Screening for Depression in Medical Settings with the Patient Health Questionnaire (PHQ): A Diagnostic Meta-Analysis 22: 1596-1602.

22. Helmreich I, Wagner S, König J, Kohnen R, Szegedi A, et al. (2015) Hamilton depression rating subscales to predict antidepressant treatment outcome in the early course of treatment. J Affect Disord 175: 199-208.

23. Schwartz TL, Siddiqui UA, Stahl SM (2011) Vilazodone: a brief pharmacological and clinical review of the novel serotonin partial agonist and reuptake inhibitor. Ther Adv Psychopharmacol 3: 81-87.

24. Hellerstein DJ, Flaxer J (2015) Vilazodone for the treatment of major depressive disorder: an evidence-based review of its place in therapy. Core Evid 10: 49-62.

25. Stahl SM (2014) Mechanism of action of the SPARI vilazodone: serotonin $1 \mathrm{~A}$ partial agonist and reuptake inhibitor. CNS Spectr 19: 105-109.

26. Feighner JP (1999) Mechanism of action of antidepressant medications. J Clin Psychiatry 60: 4-11.

27. Khan A, Cutler AJ, Kajdasz DK, Gallipoli S, Athanasiou M, et al. (2011) A randomized, double-blind, placebo-controlled, 8-week study of vilazodone, a serotonergic agent for the treatment of major depressive disorder. J Clin Psychiatry 72: 441-447.

28. Rickels K, Athanasious M, Robinson DS, Gibertini M, Whalen H, et al. (2009) Evidence for efficacy and tolerability of vilazodone in the treatment of major depressive disorder: a randomized, double-blind, placebo-controlled trial. J Clin Psychiatry 70: 326-333.

29. Viibryd (vilazodone) (2015) package insert. St. Louis, MO; Forest Pharmaceuticals, Inc.

30. Greenberg PE, Kessler RC, Birnbaum HG, Leong SA, Lowe SW, et al. (2003) The economic burden of depression in the United States: how did it change between 1990 and 2000? J Clin Psychiatry 64: 1465-1475.

31. Austin MP, Priest SR (2005) Clinical issues in perinatal mental health: new developments in the detection and treatment of perinatal mood and anxiety disorders. Acta Psychiatr Scand 112: 97-104.
Citation: Khouzam HR (2015) Vilazodone for the Treatment of Paternal Post Natal Depression. Clin Depress 1: 104.

\section{OMICS International: Publication Benefits \& Features}

\section{Unique features:}

- Increased global visibility of articles through worldwide distribution and indexing

- Showcasing recent research output in a timely and updated manner

- Special issues on the current trends of scientific research

Special features:

- 700 Open Access Journals

50,000 editorial team

Rapid review proces

Quality and quick editorial, review and publication processing

- Indexing at PubMed (partial), Scopus, EBSCO, Index Copernicus, Google Scholar etc.

Sharing Option: Social Networking Enabled

- Authors, Reviewers and Editors rewarded with online Scientific Credits

- Better discount for your subsequent articles

Submit your manuscript at: http://www.editorialmanager.com/medicaljournals/default.aspx 